\begin{tabular}{lcccc}
\hline & Baseline & 1st week & 2nd week & 1st month \\
\hline OCT (microns), mean \pm SD & $415.7 \pm 177.15$ & $413.3 \pm 162.9^{*}$ & $388.06 \pm 158.1^{*}$ & $330.8 \pm 104.2^{*}$ \\
Visual acuity, mean $\pm S D$ & $0.39 \pm 0.31$ & $0.4 \pm 0.31$ & $0.45 \pm 0.3^{*}$ & $0.51 \pm 0.3^{*}$ \\
Anterior chamber cells, median [IQR] & $1[0-1]^{*}$ & $0.5[0-1]^{*}$ & $0[0-1]^{*}$ & $0[0-0]^{*}$ \\
Vitritis, median [IQR] & $1[0-2]$ & $1[0-1.5]$ & $0[0-1]^{*}$ & $0[0-0.5]^{*}$ \\
\hline
\end{tabular}

${ }^{*} \mathrm{p}<0.05$ compared with basal data.

etanercept (2), golimumab (2), rituximab (2), abatacept (3), anakinra (1) and daclizumab (1).

TCZ administration schedule was $8 \mathrm{mg} / \mathrm{kg} / 4$ weeks iv. $(n=23)$, every 2 weeks (1) and subcutaneously $162 \mathrm{mg} / 2$ weeks (1). TCZ was used in monotherapy (13) or combined with conventional immunosuppressive drugs (12). Most of intraocular inflammation parameters showed a rapid improvement after TCZ onset (Table), with corticosteroid-sparing effect $(15.9 \pm 13.6$ to $8.5 \pm 5.17 \mathrm{mg} ; \mathrm{p}=0.001)$. Remission was achieved in 8 patients and improvement in 17. After one month of therapy, no side effects were observed.

Conclusions: TCZ seems a rapid effective treatment in refractory uveitic CME.

Disclosure of Interest: None declared

DOI: 10.1136/annrheumdis-2017-eular.5215

\section{THU0568 PREVALENCE AND AUTOIMMUNE RHEUMATIC DISEASE IN PATIENTS WITH AUTOIMMUNE/INFLAMMATORY SYNDROME INDUCED BY ADJUVANTS ASSOCIATED TO SILICONE BREAST IMPLANT}

O. Vera Lastra, N.E. Torres-Oliva, G. Medrano-Rodriguez,

M.D.P. Cruz-Dominguez, J. Sepulveda Delgado, L.J. Jara, G. Medina. Internal

Medicine, Hospital Especialidades CMN la Raza. Instituto Mexicano Seguro

Social, Mexico City, Mexico

Background: Autoimmune/inflammatory syndrome induced by adjuvants (ASIA) has been associated with previous exposure to various agents such as silicone implants, which elicit chronic stimulation of the immune system against the prosthetic material and clinical manifestation of autoimmune disease. This is particularly the case in genetically susceptible hosts.

Objectives: The aim is to describe de prevalence, family background and main autoimmune rheumatic disease (ARD) associated to silicone breast implant (SBI). Methods:

We study a cohort of 150 patients with diagnosis of ASIA associated injection of mineral oil and silicone breast implant (SBI) in a tertiary Hospital, from 2011 to 2016. All patients were evaluated for the fulfilment of ASIA criteria. We only included patients with ASIA criteria associated with SBI plus criteria for an autoimmune rheumatic disease according to The American College of Rheumatology or EULAR. We excluded patient with ASIA and without ARD.

Results: There were 17 women patients with mean age $42.4 \pm 15.3$ years, mean disease duration of disease $8 \pm 3$. The clinical manifestation post SBI appeared $8 \pm 2$ years later.

The ARD were systemic sclerosis (SSc) 5 , systemic lupus erythematosus (SLE) 3, rheumatoid arthritis (AR) 3, overlap syndrome 2 (SSc plus SS and SLE plus SSc, Sjogren syndrome 1, Takayasu arteritis 1, Still disease 1, antiphospholipid syndrome 1, and 3 patients also had secondary fibromyalgia. Five Patients had more than 2 autoantibodies, 4 patients had relatives with an ARD. All patients are being treated according to the ARD (steroids plus immunosuppressive 8 patients, immunosuppressive 7 , and only steroids 2 ), in 4 patients the prosthesis were withdrawn with improvement of clinical manifestations.

Conclusions: We found a prevalence of ASIA associated to SBI of $11 \%$. The main ARD were SSc, SLE and RA. In these cases of ASIA associated with SBI some had genetic predisposition to ARD. The use of $S B I$ is not recommended in women who have a family history of ARD.

\section{References:}

[1] Colaris MJ et al. Two hundred cases of ASIA syndrome following silicone implants: a comparative study of 30 years and rewiew of current literature. Immunol Res 2016 Jul 13

[2] Jara LJ, et al. Severe manifestation of autoimmune syndrome induced by adjuvants (Shoenfeld's syndrome) Immunol Res Jul 13.

[3] Watad et a. Autoimmune/Inflammatory induced by adjuvants (Shoenfeld's syndrome) UN update. Lupus 2017 Jan 1.

Disclosure of Interest: None declared

DOI: 10.1136/annrheumdis-2017-eular.5588

\section{THU0569 TREATMENTS OF UVEITIS IN A REFERRAL} \section{MULTIDISCIPLINARY UNIT IN NORTHERN SPAIN}

P. Fanlo ${ }^{1}$, H. Heras ${ }^{2}$, O. Terry ${ }^{1}$, G. Espinosa ${ }^{3}$, A. Adan ${ }^{4} .{ }^{1}$ Autoimmune Sistemic Disease Unit; ${ }^{2}$ Ophthalmology, Complejo Hospitalario de Navarra, Pamplona; ${ }^{3}$ Autoimmune Sistemic Disease Unit; ${ }^{4}$ Ophthalmology, Hospital Clinic, Barcelona, Spain

Background: Intraocular inflammation is one of the leading causes of visual impairment and blindness. Early and appropiate treatment is mandatory for avoiding complications.

Objectives: To describe the treatments prescribed in a cohort of patients with uveitis in a referral multidisciplinary unit from northern Spain.
Methods: Retrospective analysis of clinical records of patients evaluated in the Uveitis Multidisciplinary Unit of the Complejo Hospitalario of Navarra since January 2010 until March 2015. We analyzed the demographic characteristics and treatments received in the following 3 months after first visit.

Results: We identified 500 patients, $50 \%$ women with a mean age of $47.9+/-16.4$ years. The most frequent type of uveitis was anterior uveitis $(65,4 \%)$, followed by posterior uveitis $(17,6 \%)$, panuveitis $(15,2 \%)$, and intermediate uveitis $(1,8 \%)$. Considering the etiology, $31.2 \%$ were unclassifiable, followed by non-infectious systemic disease in $29.2 \%$.During the 3-month follow-up, 904 treatments were prescribed. The most frequent treatment was ocular topical $(39 \%)$, followed by immunosuppressive treatment (27\%), antimicrobial (14\%), other treatments (10\%) and less biological $(3 \%)$, surgical $(3 \%)$ and finally periocular $(2 \%)$ and intravitreal $(2 \%)$ treatment.Topical ocular treatment: 350 patients received topical ocular treatment, which accounts for $70 \%$ of patients. Among topical ocular treatments, $15 \%$ of the samples were treated with topical steroids, $54 \%$ were topical steroids associated with another topical treatment, $2 \%$ were topical antiglaucomatous, $2 \%$ received other topical treatments and $27 \%$ of the sample did not receive topical treatment.Immunosuppressive treatment: 249 immunosuppressive treatments were prescribed. $50 \%$ of the patients received immunosuppressive treatment. Among the immunosuppressive treatments, $25 \%$ of the patients received oral steroids, $6 \%$ salazopyrine, $4 \%$ methotrexate, $5 \%$ azathioprine, $2 \%$ mycophenolate mofetil, $5 \%$ oral steroids associated with another immunosuppressant, $15 \%$ salazopyrine associated with another immunosuppressant, $1 \%$ other immunosuppressive treatment and $49 \%$ of patients did not receive any immunosuppressive treatment.Biological Treatments: 25 patients in the cohort received biological treatment, this represents $5 \%$ of patients. The biological treatment types were distributed as follows: $3 \%$ of the patients received adalimumab treatment, $1 \%$ received infliximab, $1 \%$ received other biological treatments, and $95 \%$ of the patients did not receive biological treatments. The number of treatments received per patient was analyzed and 50 patients (10\%) received no treatment, 152 patients (30\%) received 1 treatment, 189 patients $(38 \%)$ received 2 treatments, 75 patients (15\%) Had received 3 treatments, 23 patients $(5 \%)$ had received 4 treatments, 9 patients $(2 \%)$ had received 5 treatments and lastly 2 patients had received 6 treatments.

Conclusions: The majority of patients received the combination of two treatments. Topical steroids and oral steroids were the most frequent treatments used.

Disclosure of Interest: None declared

DOI: 10.1136/annrheumdis-2017-eular.1937

\section{THU0570 ANAKINRA AS A SUCCESSFUL TREATMENT OF IDIOPATHIC RECURRENT PERICARDITIS: TAPER OR NOT TO TAPER? CASE SERIES AT THE UNIVERSITY OF SOUTHERN CALIFORNIA}

P.S. Chhibar, G. Ehresmann. Rheumatology, University of Southern California, Los Angeles, United States

Background: Idiopathic Recurrent Pericarditis can be challenging to treat in patients unresponsive to NSAIDs, aspirin, colchicine and immunosuppressive drugs. Patients become steroid dependent and tapering precipitates recurrences. Objectives: Report 2 adult cases of idiopathic recurrent pericarditis treated successfully with Anakinra.

Methods: Chart review of 2 patients with idiopathic recurrent pericarditis treated with anakinra at the Keck Medical Center of USC. Literature review on treatment of idiopathic recurrent pericarditis with anakinra.

Results: Case 1: 60-year-old Caucasian male had five episodes of idiopathic pericarditis in 2011. Serologic workup including ANA, anti-dsDNA, malignant and infectious workup was negative. Initially, patient responded to prednisone 0.4 $\mathrm{mg} / \mathrm{kg} /$ day. Adding colchicine, azathioprine and methotrexate failed to prevent recurrence. Pericarditis developed whenever prednisone was tapered below 20 $\mathrm{mg} /$ day with bursts of CRP to $78 \mathrm{mg} / \mathrm{dl}$. In 2012, Anakinra $100 \mathrm{mg}$ sq daily resulted in immediate clinical response and normalization of CRP $(1 \mathrm{mg} / \mathrm{dl})$. Prednisone and methotrexate were tapered with no recurrence. Gradually Anakinra was tapered to 3 times/week, then once a week, with no recurrence. Case 2: 37-yearold African American male had four episodes of recurrent pericarditis. He had positive ANA 1:320, but negative anti-dsDNA, anti-smith, negative infectious and malignancy workup. Initially, patient responded to prednisone $0.6 \mathrm{mg} / \mathrm{kg} / \mathrm{day}$ and colchicine. Tapering steroids below $40 \mathrm{mg} /$ day resulted in recurrent pericarditis. Sequential addition of hydroxychloroquine, methotrexate, mycophenolate mofetil, and azathioprine failed to prevent recurrence. Anakinra $100 \mathrm{mg}$ sq daily resulted in prompt resolution of symptoms, normalization of acute phase reactants and allowed successful tapering of steroids. Anakinra is being slowly tapered over the past year, with no recurrence.

Conclusions: Idiopathic recurrent pericarditis, which requires chronic corticosteroids, should be treated by adding another immunosuppressive agent. European Society of Cardiology guidelines recommend azathioprine, cyclophosphamide, methotrexate, hydroxychloroquine, cyclosporine or mycophenolate mofetil. Anakinra has demonstrated success in treating autoinflammatory and autoimmune diseases including FMF, TNF receptor associated periodic syndrome, rheumatoid arthritis and in patients with TRAPS mutation TNFRSF1A. This represented the possibility to decrease inflammation by blocking interleukin-1. Using this rationale we treated our patients. Picco et al reported three pediatric cases treated with anakinra, where its discontinuation resulted in recurrence. 
Vassilopoulos et al reported three adult cases, where two were not treated with immunosuppressive drugs before anakinra and one had recurrence after anakinra was tapered. The Double Blind Placebo Controlled Clinical Trial AIRTRIP, shows efficacy of anakinra in treating 11 patients with recurrent pericarditis over 14 months. It is unclear from this study if anakinra should be tapered or not. We suggest that once steroids and immunosuppressive drugs have been discontinued, anakinra should be gradually tapered over months, to avoid relapse. These experiences warrant further long term controlled trials in order to determine the efficacy and appropriate treatment regimen of anakinra for recurrent pericarditis.

Disclosure of Interest: None declared

DOI: 10.1136/annrheumdis-2017-eular.1141

\section{THU0571 THE CLINICAL FEATURES OF 223 BEHCET'S DISEASE PATIENTS IN JAPAN}

R. Saito ${ }^{1}$, K. Nishimura ${ }^{1}$, H. Mukoyama ${ }^{1}$, Y. Nakamura ${ }^{1}$, T. Nagamoto ${ }^{1}$, K. Akashi ${ }^{2}$, A. Onishi ${ }^{2}$, Y. Kogata ${ }^{2}$, J. Saegusa ${ }^{2}$, A. Morinobu ${ }^{2}$, T. Yokota ${ }^{1}$. ${ }^{1}$ Department of Endocrinology and Rheumatology, Kurashiki Central Hospital, Kurashiki; ${ }^{2}$ Department of Rheumatology and Clinical Immunology, Kobe University Graduate School of Medicine, Kobe, Japan

Background: Behcet's disease is a systemic vasculitis disease with oral and genital aphthous ulcers, ocular involvements, skin manifestations, arthritis, gastrointestinal manifestations, neurogenic diseases and vascular involvements. Patients with Behcet's disease are known to distribute along the ancient Silk Road, including Japan.

Objectives: We evaluate the clinical features of Behcet's disease in Japan. Methods: We retrospectively investigated 223 patients (108 males and 115 females) who fulfilled the International Criteria for Behcet's Disease (ICBD) from January, 2006 until May, 2015. We examined sex, onset age, disease type, clinical symptoms, laboratory data and medications.

\section{Results:}

Median age at diagnosis was $36.0 \pm 12.8$ years old. Oral ulcers were the most common manifestation $(98.2 \%)$, followed by genital ulcers $(62.4 \%)$, ocular involvements $(53.2 \%)$, erythema nodosum $(53.2 \%)$, acneiform lesions $(51.8 \%)$, arthritis $(38.6 \%)$, gastrointestinal manifestations $(25.1 \%)$, neurogenic diseases $(9.0 \%)$, and vascular involvements $(8.1 \%)$. The relationship of HLA and disease manifestations was studied in 123 patients (41.5\% with HLA-B51 and $24.1 \%$ with HLA-A26). The frequency of acneiform lesions, ocular involvements and HLA-B51 was significantly higher in male, while genital ulcers and arthritis were significantly higher in female. Patients with ocular involvements showed a higher assosiation rate with neurogenic diseases and HLA-B51, and lower wih gastrointestinal manifestations. TNF $\alpha$ inhibitor (infliximab or adalimumab) were used for 66 cases $(30 \%)$, and it could be continued for 1 year in $91 \%$, and for 2 years in $83 \%$.

\begin{tabular}{|c|c|c|c|c|}
\hline & Total $(n=223)$ & Male $(n=108)$ & Female $(n=115)$ & $\mathrm{pva}$ \\
\hline Oral ulcers & $218 / 222(98.2 \%)$ & $105 / 107(98.1 \%)$ & $113 / 115(98.3 \%)$ & 0.94 \\
\hline Skin manifestations & 190/220 (86.4\%) & 90/107 (84.1\%) & 100/113 (88.5\%) & 0.34 \\
\hline Erythema nodosum & $116 / 218(53.2 \%)$ & $49 / 105(46.7 \%)$ & $67 / 113(59.3 \%)$ & 0.06 \\
\hline Acneiform lesions & $113 / 218(51.8 \%)$ & $65 / 105(61.9 \%)$ & $48 / 113(42.5 \%)$ & 0.004 \\
\hline Thrombophlebitis & $11 / 218(5.0 \%)$ & $5 / 105(4.8 \%)$ & $6 / 113(5.3 \%)$ & 0.85 \\
\hline Ocular involvements & $118 / 222(53.2 \%)$ & $68 / 108(63.0 \%)$ & $50 / 114(43.9 \%)$ & 0.004 \\
\hline Genital ulcers & $138 / 221(62.4 \%)$ & $58 / 107(54.2 \%)$ & 80/114 (70.2\%) & 0.01 \\
\hline Artiritis & $86 / 223(38.6 \%)$ & $33 / 108(30.6 \%)$ & $53 / 115(46.1 \%)$ & 0.02 \\
\hline Gastrointestinal manifestations & $56 / 223(25.1 \%)$ & $23 / 108(21.3 \%)$ & $33 / 115(28.7 \%)$ & 0.20 \\
\hline Neurogenic diseases & $20 / 223(9.0 \%)$ & $12 / 108(11.1 \%)$ & $8 / 115(7.0 \%)$ & 0.28 \\
\hline Vascula & $18 / 223(8.1 \%)$ & $6 / 108(5.6 \%)$ & $12 / 115(10.4 \%)$ & 0.18 \\
\hline HLA-B5 & $51 / 123(41.5 \%)$ & $32 / 62(51.6 \%)$ & $19 / 61(31.1 \%)$ & 0.02 \\
\hline HLA-A2 & $28 / 116(24.1 \%)$ & $14 / 56(25.0 \%)$ & $14 / 60(23.3 \%)$ & 0.91 \\
\hline
\end{tabular}

\begin{tabular}{lcccc} 
Table 2 & \multicolumn{5}{l}{} & \\
\hline & $\begin{array}{c}\text { Total } \\
(\mathrm{n}=223)\end{array}$ & $\begin{array}{c}\text { With ocular } \\
\text { involvements }(\mathrm{n}=118)\end{array}$ & $\begin{array}{c}\text { Without ocular } \\
\text { involvements }(\mathrm{n}=104)\end{array}$ & $\mathrm{p}$ value \\
\hline Gastrointestinal & & & & \\
$\quad$ manifestations & $56 / 223(25.1 \%)$ & $17 / 118(14.4 \%)$ & $39 / 104(37.5 \%)$ & $<0.001$ \\
Neurogenic diseases & $20 / 223(9.0 \%)$ & $19 / 118(16.1 \%)$ & $1 / 104(1.0 \%)$ & $<0.001$ \\
Vascular involvements & $18 / 223(8.1 \%)$ & $7 / 118(5.9 \%)$ & $11 / 104(10 \% \%)$ & 0.21 \\
HLA-B51 positive & $51 / 123(41.5 \%)$ & $36 / 69(52.2 \%)$ & $15 / 54(27.8 \%)$ & 0.006 \\
HLA-A26 positive & $28 / 116(24.1 \%)$ & $19 / 65(29.2 \%)$ & $9 / 51(17.6 \%)$ & 0.15 \\
\hline
\end{tabular}

Conclusions: A higher incidence of gastrointestinal manifestations was observed in patients with Behcet's disease in Japan. Patients with ocular involvements showed a higher assosiation rate with neurogenic diseases, and lower wih gastrointestinal manifestation. Most patients could continue TNF $\alpha$ inhibitor safety and effectively.

Disclosure of Interest: None declared

DOI: 10.1136/annrheumdis-2017-eular.4216

\section{THU0572 ASSOCIATION BETWEEN RETROPERITONEAL FIBROSIS AND MALIGNANCY: A POSSIBLE PARANEOPLASTIC SYNDROME}

S.J. Lee ${ }^{1}$, J.S. Eun ${ }^{1}$, E.Y. Lee ${ }^{2}$, G.B. Bae ${ }^{1}$, E.J. Nam ${ }^{1}$, Y.W. Song ${ }^{2}$, Y.M. Kang ${ }^{1} .{ }^{1}$ Internal Medicine, Division of Rheumatology, Kyungpook National University Hospital, Daegu; ${ }^{2}$ Internal Medicine, Division of Rheumatology, Seoul National University Hospital, Seoul, Korea, Republic Of

Background: Retroperitoneal fibrosis (RPF) are associated with malignancies. However it is unclear what is the incidence of malignancies and whether particular malignancies are more prevalent in RPF.

Objectives: The objective of this study was to examine standardized incidence ratios (SIRs) of cancers in patients with retroperitoneal fibrosis (RPF) compared with age- and sex-matched general population.

Methods: Medical records of 111 patients diagnosed as having RPF by computed tomography, positron emission tomography and/or histological evaluation were reviewed. Forty one cases of cancers, which were confirmed by biopsies, were identified in 35 patients with RPF. SIRs were calculated for cancers, cancer types, and age at cancer diagnosis and stratified according to RPF-cancer intervals compared with general population in Korea.

Results: The mean \pm SD age at RPF diagnosis was $59.1 \pm 14.9$ years, and $69.4 \%$ of the patients were male. The cancer SIR (95\% confidence intervals) in patients with RPF relative to age- and sex-matched individuals in the general population was $3.18(2.23$ - 4.41) [2.65 (1.7 - 3.94) in men; 5.34 (2.76 - 9.32) in women]. The most frequent cancer was unspecified urinary organ cancers with SIR of 733.41 (238.14 - 1711.53). SIRs of multiple myeloma [27.58 (3.34 - 99.64)], renal cell cancers [9.53, (1.15 - 34.42)] and unspecified cancers [16.92, (2.05 - 61.12)] were also significantly higher than in general population. Whereas cancers were most frequently developed in the eighth decade of life, the peak SIR was observed in the fifth decade $(8.41,2.29-21.53)$. When stratified by RPF-cancer intervals, SIR was 6.85 (4.55 - 9.90) within 2 years of RPF diagnosis, while no significant increase in SIR was found out of 2 years. Malignancies $(n=28)$ within 2 year of RPF diagnosis included unspecified urinary organ cancer $(n=4)$, stomach cancer $(n=4)$, lung cancer $(n=4)$, colon cancer $(n=3)$, renal cell cancer $(n=2)$, pancreatic cancer $(n=2)$, unspecified cancer $(n=2)$, rectal cancer $(n=1)$, gallbladder cancer $(n=1)$, non-Hodgkin lymphoma $(n=1)$, multiple myeloma $(n=1)$, prostate cancer $(n=1)$, thyroid cancer $(n=1)$ and gastrointestinal stromal tumor $(n=1)$. Predominant origin of these malignancies were epithelial cell types [transitional cell carcinomas $(n=4)$, adenocarcinoma $(n=16)]$.

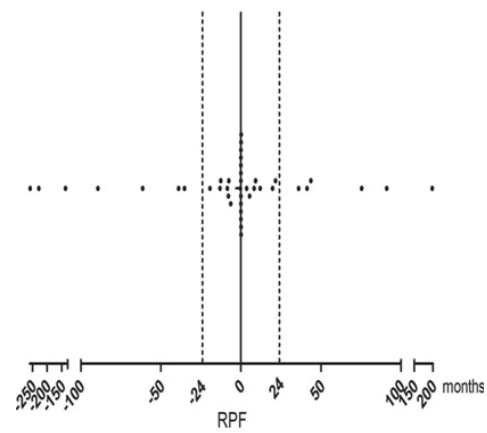

Conclusions: RPF was strongly associated with cancers, particularly within 2 years of RPF diagnosis. Our results indicate that cancer screening in patients with RPF should may be performed regularly up to 2 years after RPF diagnosis. References:

[1] Temporal relationship between cancer and myositis identifies two distinctive subgroups of cancers: impact on cancer risk and survival in patients with myositis. Kang EH, Lee SJ et al Rheumatology (Oxford). 2016 Sep;55(9):163141.

Disclosure of Interest: None declared

DOI: 10.1136/annrheumdis-2017-eular.6168

\section{THU0573 CONCOMITANT AUTOIMMUNE DISEASES IN PATIENTS WITH SARCOIDOSIS}

S. Kobak ${ }^{1}$, F. Sever ${ }^{2}$, H. Semiz ${ }^{3}$, M. Orman ${ }^{4} .{ }^{1}$ Rheumatology, Istinye University Faculty of Medicine, LIV Hospital, Istanbul; ${ }^{2}$ Chest Diseases, Medicalpark Hospital; ${ }^{3}$ Internal Medicine; ${ }^{4}$ Statistics, Ege University Faculty of Medicine, Izmir, Turkey

Background: Sarcoidosis is a chronic granulomatous disease characterized by non-caseating granuloma formation. It can mimic many rheumatic diseases and/or may be coexist with them. There are limited data in the literature about the association of sarcoidosis with autoimmune diseases.

Objectives: The purpose of this study is to determine the frequency and characteristics of autoimmune diseases associated with patients with sarcoidosis. Methods: One hundred and thirty-one sarcoidosis patients folowed-up in single rheumatology center were included in the study. Demographic, clinical, laboratory and radiological data of these cases were evaluated retrospectively. The 